
\title{
R Research S Surare \\ Environmental exposure and clinical correlates of hepatocellular carcinoma in New York City: A case only study
}

Hui-Chen Wu ( $\sim$ hw2057@cumc.columbia.edu )

Columbia University Medical Center: Columbia University Irving Medical Center Jing Shen

Rutgers Robert Wood Johnson Medical School Camden: Rutgers Robert Wood Johnson Medical School Abby Siegel

Merck \&amp; Co Inc

Regina M. Santella

Columbia University Medical Center: Columbia University Irving Medical Center

\section{Short Report}

Keywords: Hepatocellular carcinoma (HCC), environmental exposure, clinical correlates, New York City

Posted Date: April 15th, 2021

DOI: https://doi.org/10.21203/rs.3.rs-249569/v1

License: (9) This work is licensed under a Creative Commons Attribution 4.0 International License. Read Full License

Version of Record: A version of this preprint was published at Cancer Causes \& Control on September 8th, 2021. See the published version at https://doi.org/10.1007/s10552-021-01494-2. 


\section{Abstract}

In the U.S., Hepatocellular carcinoma (HCC) incidence rates have increased. We aimed to determine whether environmental exposure plays a role in the high incidence of HCC observed in New York City. We conducted a hospital-based case only study to examine the prevalence of aflatoxin B1 (AFB1)- and polycyclic aromatic hydrocarbon (PAH)-albumin adducts and the distribution of adducts by different characteristics of HCC patients. Blood samples were collected from 155 HCC patients for biomarker analyses. We observed that about $46 \%$ and $49 \%$ of cases had detectable AFB1- and PAH-albumin adducts, respectively. There were significant differences between AFB1-albumin adducts and selected factors such as HCV infection status $(p=0.04)$, diabetes $(p=0.03)$ and Barcelona Clinic Liver Cancer (BCLC) stage $(p=0.02)$. Cases with detectable PAH-albumin adducts had a smoking history compared with those with nondetectable levels $(p=0.04)$. The level of AFB1-albumin adducts was positively correlated with plasma bilirubin $\left(r_{\mathrm{s}}=0.32, p<0.0001\right)$ and adiponectin concentrations $\left(r_{\mathrm{s}}=0.28, p=\right.$ 0.0005). The level of aflatoxin B1-albumin adducts was negatively associated with blood albumin concentration $\left(r_{\mathrm{s}}=-0.28, p=0.0009\right)$ and plasma DNA LINE-1 methylation $\left(r_{\mathrm{s}}=-0.16, p=0.04\right)$. Our study provides additional evidence that environmental exposures including to aflatoxin might partially explain the increase in the incidence of HCC in the US.

\section{Introduction}

Hepatocellular carcinoma (HCC) death rates are rising, even as most other cancers in the US are declining. ${ }^{1}$ In New York City (NYC), HCC is one of the leading causes of cancer-related death. The risk of dying from HCC is much greater for people who live in the Bronx and nearby neighborhoods that are predominantly populated by Blacks and Hispanics, compared to other parts of NYC and the US. ${ }^{2}$ Differences in traditional risk factors likely contribute to HCC disparity, ${ }^{2}$ but less is known about the role of environmental exposure in both geographic and racial disparities.

Aflatoxins, genotoxic hepatocarcinogens, are toxic fungal metabolites that commonly contaminate crops. Polycyclic aromatic hydrocarbons (PAHs), possible human carcinogens, are ubiquitous pollutants caused by incomplete combustion of various materials, including diesel fuel and tobacco. ${ }^{3}$ Using a prospective study design, we reported that higher levels of aflatoxin B1- (AFB1) and PAH-albumin adducts were associated with HCC risk in Taiwan where the incidence of HCC is high. ${ }^{4}$ A recent survey of 184 volunteers recruited in a Hispanic community in South Texas where the incidence of HCC is high reported aflatoxin adducts were detected in $21 \%$ of the participants with a mean level 5 -fold higher than that detected in the US general population. ${ }^{5}$ These observation suggest that aflatoxin might explain some of the epidemiology of US HCC in term of the significant geographic and ethnic variations in incidence. ${ }^{1}$

Here, we conducted a hospital-based case only study to examine the prevalence of AFB1- and PAHalbumin adducts and the distribution of adducts by different characteristics of HCC patients that were 
treated in a medical center located in the heart of the Washington Heights neighborhood of northern Manhattan.

\section{Materials And Methods Patients}

This study used information from a total of 155 adult patients ( $\geq 18$ years old) with newly diagnosed HCC and treated in the Hepatobiliary Oncology Clinics at the Columbia University Irving Medical Center (CUIMC) during October 2008 to July 2014. Subjects completed an epidemiologic questionnaire, underwent a physical examination (including weight and height measurements) and provided a fasting morning blood sample at the time of enrollment. Information on biochemical blood analyses, including viral hepatitis, albumin, and total bilirubin, were obtained from the medical records. Simultaneously, an additional $30 \mathrm{~mL}$ of blood was processed to isolate plasma and buffy coat which were frozen at $-80^{\circ} \mathrm{C}$. This study was approved by the CUIMC Institutional Review Board.

\section{Aflatoxin B1- and PAH-albumin adduct measurement}

We used ELISA assay for aflatoxin-and PAH-albumin adducts. ${ }^{4}$ Briefly, $50 \mu \mathrm{l}$ of albumin extracts, equivalent to $200 \mathrm{\mu g}$ of albumin, were added to 96 -well plates (Easywash, Corning, NY) previously coated with $5 \mathrm{ng}$ of 7ß,8a-dihydroxy-9a,10a-epoxy-7,8,9,10-tetrahydrobenzo(a)pyrene (BPDE-I)-DNA. The standard curve was generated by adding serial dilutions of BPDE-I-tetrols in PBS from 5 to $2500 \mathrm{fmol}$ in $50 \mu$ into the coated wells followed by $50 \mu$ l of antibody. The values for each subject were expressed as $\mathrm{fmol}$ of PAH per mg albumin. Samples with less than $15 \%$ inhibition were considered undetectable and assigned a value of $50 \mathrm{fmol} / \mathrm{mg}$ albumin.

For $\mathrm{AFB}_{1}$-albumin adduct were assayed by ELISA: enzyme digested albumin was extracted and the

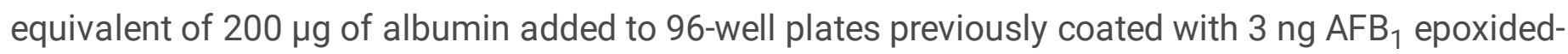
modified human serum albumin. Polyclonal antiserum 7 was used and samples with $<20 \%$ inhibition were considered non-detectable and assigned a value of $4.5 \mathrm{fmol} / \mathrm{mg}$.

\section{Measurement of LINE-1 methylation}

Plasma DNAs were isolated using QIAmp UltraSense Virus Kits (Qiagen, Valencia, CA). Aliquots of DNA (500 ng) were bisulfite-treated with the EZ DNA methylation kit (Zymo Research, Orange, CA) following the manufacturer's protocol. Pyrosequencing for LINE-1 methylation levels was carried out using PCR and sequencing primers as described previously. ${ }^{5}$ Briefly, PCR was carried out in a $25 \mu$ reaction mix containing 50 ng bisulfite-converted DNA, 1X Pyromark PCR Master Mix (Qiagen), 1X Coral Load Concentrate (Qiagen) and $0.2 \mu \mathrm{M}$ forward and reverse primers. Following amplification, the biotinylated PCR products were purified and incubated with the sequencing primer designed to bind adjacent to the CpG sites of interest. Pyrosequencing was conducted using a PyroMark Q96 instrument (Qiagen), with subsequent quantitation of methylation levels determined with the PyroMark $\mathrm{CpG} 1.010$ software. 
Relative peak height differences were used to calculate the percentage of methylated cytosines at each given site. Percent methylation within a sample was subsequently determined by averaging across all three interrogated $\mathrm{CpG}$ sites in the analysis.

Plasma adiponectin measurement.

Human adiponectin levels was determined in duplicate using commercial ELISA Kits (ThermoFisher Scientific, Camarillo, CA, USA). A serial dilution of adiponectin (from 0.5 to $32 \mathrm{ng} / \mathrm{ml}$ ) was used to generate a standard curve.

\section{Statistical Analysis}

We used the $\chi^{2}$ test for categorical variables and Student's $t$-test for continuous variables to assess the difference in selected characteristics between exposure groups (detectable vs undetectable adducts). We calculated the Spearman rank correlation coefficients $\left(r_{s}\right)$ to determine the correlation of adducts and plasma LINE-1 methylation. All analyses were performed with SAS software 9.4 (SAS Institute, Cary, NC).

\section{Results}

The racial distribution of our HCC cases is diverse and includes $48 \%$ whites, $25 \%$ of Hispanics, $13 \%$ Asian and $10 \%$ Blacks. There were about $46 \%$ and $49 \%$ of cases with detectable AFB1- and PAH-albumin adducts, respectively. The mean level was $10.8 \mathrm{fmol} / \mathrm{mg}$ for AFB1-albumin adducts and $116.9 \mathrm{fmol} / \mathrm{mg}$ for PAH-albumin adducts.

There were significant differences between AFB1-albumin adducts and selected factors such as HCV infection status $(p=0.04)$, diabetes $(p=0.03)$ and Barcelona Clinic Liver Cancer (BCLC) stage $(p=0.02)$ (Table 1). Cases with detectable PAH-albumin adducts were older $(p=0.02)$ and had smoking history compared with those with nondetectable levels $(p=0.04)$. 
Table 1

The distribution of aflatoxin B1 (AFB1)- and polycyclic aromatic hydrocarbon (PAH)-albumin adducts by selected characteristics among hepatocellular carcinoma cases

\section{AFB1-albumin adducts \\ PAH-albumin adducts}

Detectable Undetctable $\begin{aligned} & \mathrm{P} \\ & \text { value }\end{aligned} \quad$ Detectable Undetctable $\begin{aligned} & \mathrm{P} \\ & \text { value }\end{aligned}$

\begin{tabular}{|c|c|c|c|c|c|c|}
\hline $\mathrm{N}(\%)$ & $\begin{array}{l}N=71 \\
(46 \%)\end{array}$ & $\begin{array}{l}N=84 \\
(54 \%)\end{array}$ & & $\begin{array}{l}N=73 \\
(49 \%)\end{array}$ & $\begin{array}{l}N=76 \\
(51 \%)\end{array}$ & \\
\hline Age, mean (SD) & $61.5(10.8)$ & $64.3(9.8)$ & 0.11 & $65.4(10.6)$ & $61.7(8.3)$ & 0.02 \\
\hline \multicolumn{7}{|l|}{ Gender } \\
\hline Female & $18(26)$ & $16(19)$ & 0.34 & $14(19)$ & $19(25)$ & 0.39 \\
\hline Male & $53(74)$ & $68(81)$ & & $59(81)$ & $58(75)$ & \\
\hline \multicolumn{7}{|l|}{ Race } \\
\hline White & $38(53)$ & $37(46)$ & 0.1 & $41(56)$ & $30(39)$ & 0.01 \\
\hline Hispanics & $14(20)$ & $25(30)$ & & $20(27)$ & $18(24)$ & \\
\hline Blacks & $11(15)$ & $5(6)$ & & $4(5)$ & $11(15)$ & \\
\hline Asian & $6(8)$ & $14(17)$ & & $7(10)$ & $13(17)$ & \\
\hline Other & $2(2)$ & $3(4)$ & & $1(1)$ & $4(5)$ & \\
\hline
\end{tabular}

Foreign Born

$\begin{array}{lllllll}\text { No } & 38(56) & 41(55) & 0.63 & 41(58) & 36(51) & 0.45 \\ \text { Yes } & 30(44) & 34(45) & & 30(42) & 34(49) & \end{array}$

Education

$\begin{array}{lcccccc}\begin{array}{l}\text { Less than high } \\ \text { school }\end{array} & 34(50) & 31(39) & 0.19 & 29(41) & 34(49) & 0.36 \\ \begin{array}{l}\text { High school and } \\ \text { above }\end{array} & 34(50) & 48(61) & & 42(59) & 36(51)\end{array}$

Annual income

$\begin{array}{lllllll}<\$ 30000 & 21(37) & 26(39) & 0.78 & 23(39) & 23(38) & 0.71 \\ \$ 30000-60000 & 14(25) & 13(19) & & 15(25) & 12(20) & \\ >\$ 60000 & 22(39) & 28(42) & & 21(36) & 25(42)\end{array}$

HCV

Negative

24 (34)

$42(50)$

$0.04 \quad 35(48)$

30 (39)

0.3 


\begin{tabular}{|c|c|c|c|c|c|c|}
\hline \multirow[b]{2}{*}{ Positive } & \multicolumn{3}{|c|}{ AFB1-albumin adducts } & \multicolumn{3}{|c|}{ PAH-albumin adducts } \\
\hline & $47(66)$ & $42(50)$ & & $38(52)$ & $46(61)$ & \\
\hline \multicolumn{7}{|l|}{ HBV } \\
\hline Negative & $62(87)$ & $65(77)$ & 0.11 & $63(86)$ & $58(76)$ & 0.12 \\
\hline Positive & $9(13)$ & $19(23)$ & & $10(14)$ & $18(24)$ & \\
\hline \multicolumn{7}{|c|}{ Alcohol Use } \\
\hline Never & $27(38)$ & $29(34)$ & 0.65 & $23(31)$ & $31(41)$ & 0.24 \\
\hline Ever & $44(62)$ & $55(66)$ & & $50(69)$ & $45(59)$ & \\
\hline \multicolumn{7}{|c|}{ Smoking history } \\
\hline Never & $22(32)$ & $36(46)$ & 0.1 & $23(33)$ & $35(49)$ & 0.04 \\
\hline Ever & $46(68)$ & $43(54)$ & & $47(67)$ & $36(51)$ & \\
\hline \multicolumn{7}{|l|}{ Diabetes } \\
\hline No & $47(66)$ & $41(49)$ & 0.03 & $38(52)$ & $48(63)$ & 0.17 \\
\hline Yes & $24(34)$ & $43(51)$ & & $35(48)$ & $28(37)$ & \\
\hline \multicolumn{7}{|c|}{ BCLC stage } \\
\hline 0 or $\mathrm{A}$ & $20(29)$ & $38(46)$ & 0.02 & $29(40)$ & $28(37)$ & 0.76 \\
\hline$B$ or C & $50(71)$ & $45(54)$ & & $44(60)$ & $47(63)$ & \\
\hline
\end{tabular}

Figure 1 presents the statistically significant correlation of AFB1-albumin adducts and selected blood markers. There was a positive association between AFB1-albumin adducts and total bilirubin concentration, with a correlation coefficient of 0.32 . The level of AFB1-albumin adducts was negatively associated with blood albumin concentration $\left(r_{s}=-0.28, p=0.0009\right)$ and plasma DNA LINE-1 methylation $\left(r_{s}=-0.16, p=0.04\right)$. The correlation coefficient between AFB1-albumin adducts and plasma adiponectin concentration was $0.28(p=0.0005)$. No associations were found between PAH-albumin adducts and blood markers.

\section{Discussion}

Our results indicate that both aflatoxin and PAH exposures are common in US HCC cases. Although it is estimated that $0.7 \%$ of US HCC show evidence of aflatoxin exposure from the Cancer Genome Atlas analysis, ${ }^{6}$ we observed that $~ 46 \%$ of HCC cases had detectable aflatoxin adducts. Our study provides additional evidence that environmental exposure including to aflatoxin might partially explain the increase in the incidence of HCC in the US. ${ }^{5}$ 
Aflatoxin, a genotoxic hepatocarcinogen, can induce $\mathrm{G} \rightarrow \mathrm{T}$ mutations, in particular at codon $249 p 53 .^{7} \mathrm{~A}$ significant racial difference in $P 53 R 249$ S mutations, a hallmark of aflatoxin-related $\mathrm{HCC}$, in tumors from HCC cases in South Texas was also observed; The prevalence of TP53R249S mutations was highest in Asians $(72.7 \%)$, followed by Blacks (18.2\%), and Hispanics (9.1\%). ${ }^{8}$ In our study, we did not see any racial difference in AFB1-albumin adducts. However, measuring AFB1-DNA adducts and p53 mutations in HCC tumors, we previously observed that AFB1-DNA adducts were associated with p53 DNA mutation. ${ }^{7}$ As adducts serve as a biomarker of exposure, examining the mutational spectrum of tumors might assist in the better understanding of racial differences in mechanisms of AFB1 carcinogenesis.

A strong interaction between aflatoxin and HBV was reported. ${ }^{9}$ In our case-control study nested in a community-based cohort, we found that HCC risks was 6-fold higher for individuals with chronic HCV infection and low AFB1-albumin adduct, and 20 -fold for individuals with both chronic HCV infection and high adducts compared to those with low adducts and no HCV infection. ${ }^{10}$ In the present study, we observed that individuals with detectable AFB1-albumin adduct were more likely to be HCV positive. Further study is needed to better understand the molecular mechanism involved in AFB1 promoting hepatocarcinogenesis in HCV-infected liver.

We found AFB1-albumin adducts were associated with blood markers including total bilirubin, albumin, plasma LINE-1 methylation and adiponectin. Adiponectin is known to regulate inflammation, tumor microenvironment and behavior. ${ }^{11}$ A study of HCC tissues found that increased adiponectin significantly correlated with tumor size, indicating its possible role in prediction of poor prognosis. ${ }^{12}$ We previously reported lower plasma levels of LINE-1 methylation was associated with worse survival in HCC patients. ${ }^{13}$ Both animal and human studies found aflatoxin is immunosuppressive by impairing and suppressing normal immune function. ${ }^{14}$ Further study is need to understand the potential effects of aflatoxin exposure on HCC prognosis.

Analyzing plasma banked up to 12 years before diagnosis, we demonstrated a 2 -fold increased risk for development of HCC with increased PAH-albumin adducts independent of viral status. ${ }^{4}$ In the present study, we found that about half of the HCC cases had detectable PAH-albumin adducts and those with adducts were more likely to be ever smokers. Previous studies have suggested a role for smoking in development of HCC. ${ }^{15}$ That the correlation of PAH-albumin adduct and smoking suggest that PAHalbumin adducts can be used a biomarker for validating smoking cessation in HCC prevention.

Study strengths include using biomarker measurement to quantify environmental exposure at the individual level. The limitations of this study are small sample size and case only study design. A larger study will be needed to identify individuals at high risk of aflatoxin exposure which will useful for HCC prevention.

\section{Declarations}


Acknowledgements: This work was supported by awards from the National Cancer Institute [R01ES005116 and P30 CA013696] and the National Institute of Environmental Health Sciences [P30 ES009089]. The content is solely the responsibility of the authors and does not necessarily represent the official views of the $\mathrm{NIH}$.

Authorship contribution: Conceptualization (Wu, Shen, Siegel, and Santella), Data curation (Wu, Shen, Siegel, and Santella), Formal analysis (Wu), Funding acquisition (Siegel, and Santella), Investigation (Wu, Shen, Siegel, and Santella), Methodology (Wu, and Santella), Project administration (Wu and Santella), Resource (Santella), Software (Wu), Supervision (Santella), Writing-original draft (Wu), writingreview/editing (Wu, Shen, Siegel, and Santella),

Data sharing: Contact the corresponding author for any inquiries regarding data or analytical code.

\section{Disclosure of Interest:}

The authors report no conflict of interest.

\section{References}

1. White DL, Thrift AP, Kanwal F, Davila J, El-Serag HB (2017) Incidence of Hepatocellular Carcinoma in All 50 United States, From 2000 Through 2012. Gastroenterology 152(4):812-820.e815

2. Kamath GR, Taioli E, Egorova N (2018) N, et al. Liver Cancer Disparities in New York City: A Neighborhood View of Risk and Harm Reduction Factors. Front Oncol 8:220-220 :.PMC6011126

3. Phillips DH (1999) Polycyclic aromatic hydrocarbons in the diet. Mutation Research/Genetic Toxicology Environmental Mutagenesis 443(1):139-147

4. Wu H-C, Wang Q, Wang L-W et al (2007) Polycyclic Aromatic Hydrocarbon- and Aflatoxin-albumin Adducts, and Hepatitis B Virus Infection and Hepatocellular Carcinoma in Taiwan. Cancer Lett 252(1):104-114.PMC1948099

5. Johnson NM, Qian G, Xu L et al (2010) Aflatoxin and PAH exposure biomarkers in a U.S. population with a high incidence of hepatocellular carcinoma. Sci Total Environ 408(23):6027-6031

6. Huang MN, Yu W, Teoh WW et al (2017) Genome-scale mutational signatures of aflatoxin in cells, mice, and human tumors. Genome Res 27(9):1475-1486

7. Lunn RM, Zhang Y-J, Wang L-Y et al (1997) p53 Mutations, Chronic Hepatitis B Virus Infection, and Alfatoxin Exposure in Hepatocellular Carcinoma in Taiwan. Cancer Research 57(16):3471-3477

8. Jiao J, Niu W, Wang Y, et al. Prevalence of Aflatoxin-Associated $<$ em $>$ TP53R249S $</$ em $>$ Mutation in Hepatocellular Carcinoma in Hispanics in South Texas. Cancer Prevention Research. 2018;11(2):103-112

9. Wu H-C, Wang Q, Yang H-l et al (2009) Aflatoxin B(1) Exposure, Hepatitis B Virus Infection and Hepatocellular Carcinoma in Taiwan. Cancer epidemiology biomarkers prevention 18(3):846853.PMC3535829 
10. Chu Y-J, Yang H-I, Wu H-C et al (2018) Aflatoxin B(1) exposure increases the risk of hepatocellular carcinoma associated with hepatitis $\mathrm{C}$ virus infection or alcohol consumption. European journal of cancer (Oxford England: 1990) 94:37-46 :.PMC5895495

11. Deng T, Lyon CJ, Bergin S, Caligiuri MA, Hsueh WA (2016) Obesity, Inflammation, and Cancer. Annu Rev Pathol 11:421-449

12. Chen MJ, Yeh YT, Lee KT, Tsai CJ, Lee HH, Wang SN (2012) The promoting effect of adiponectin in hepatocellular carcinoma. J Surg Oncol 106(2):181-187

13. Yeh C-C, Goyal A, Shen J et al (2017) Global Level of Plasma DNA Methylation is Associated with Overall Survival in Patients with Hepatocellular Carcinoma. Annals of surgical oncology 24(12):3788-3795.PMC5801698

14. Mohsenzadeh MS, Hedayati N, Riahi-Zanjani B, Karimi G (2016) Immunosuppression following dietary aflatoxin B1 exposure: a review of the existing evidence. Toxin Reviews 35(3-4):121-127

15. Petrick JL, Campbell PT, Koshiol J et al (2018) Tobacco, alcohol use and risk of hepatocellular carcinoma and intrahepatic cholangiocarcinoma: The Liver Cancer Pooling Project. Br J Cancer 118(7):1005-1012.PMC5931109

\section{Figures}



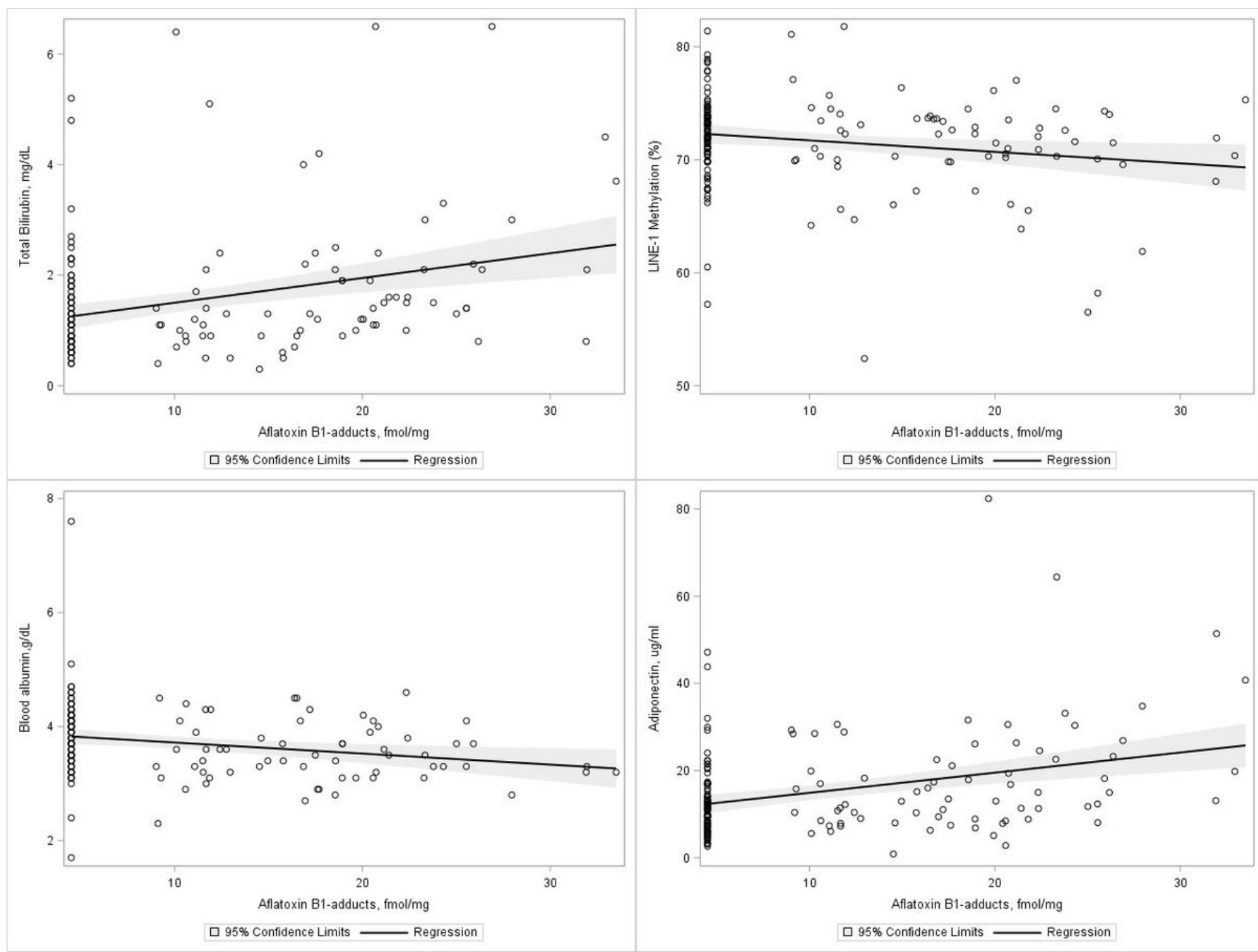

Figure 1

The correlation of aflatoxin B1-albumin adducts and blood markers. Top left panel: A. The correlation of aflatoxin B1-albumin adducts and total bilirubin concentration ( $r s=0.32, p<0.0001)$. Bottom left panel: $B$. The correlation of aflatoxin B1-albumin adducts and blood albumin concentration ( $r s=-0.28, p=0.0009)$. Top right panel: C. The correlation of aflatoxin B1-albumin adducts and plasma DNA LINE-1 methylation ( $r s=-0.16, p=0.04)$. Bottom right panel: $D$. The correlation of aflatoxin $B 1$-albumin adducts and plasma adiponectin concentration ( $r s=0.28, p=0.0005)$ 